\title{
Perfil de Urgências Odontológicas no Estado da Paraíba: Uma Análise do PMAQ-AB
}

\author{
Deborah Ellen Wanderley Gomes Freire, ${ }^{1}$ Yuri Wanderley Cavalcanti, ${ }^{2}$ \\ Aldelany Ramalho Freire, ${ }^{3}$ Edson Hilan Gomes de Lucena ${ }^{4}$
}

\begin{abstract}
RESUMO
Objetivo: Avaliar os fatores associados à procura por atendimentos de urgência odontológica na Atenção Primária à Saúde no Estado da Paraíba. Metodologia: Foi realizado estudo transversal analítico, a partir dos microdados do $1^{\circ}$ ciclo de avaliação externa do Programa de Melhoria do Acesso e da Qualidade da Atenção Básica (PMAQ-AB). Dados de relato de casos de urgência odontológica ( $n=1.531$ ) e da procura por atendimento após episódio de urgência ( $n=824)$ foram avaliados segundo características sociodemográficas (sexo, idade, estado civil, cor da pele, analfabetismo, renda e cadastro no Bolsa Família). Os dados foram analisados por meio de regressão logística multivariada, pela qual foram obtidas as medidas de razão de chances (OR) e intervalo de confiança (IC95\%), considerando-se $p<0,05$. Resultados: Indivíduos que informaram não saber ler apresentaram maior chance $(\mathrm{OR}=1,430, \mathrm{IC} 95 \%=1,113-1,836)$ de relatar alguma urgência odontológica. As variáveis idade e renda compuseram o modelo ajustado de regressão, porém sem significância $(p>0,05)$. Entre os indivíduos que relataram urgência odontológica, 57,9\% informaram procurar atendimento odontológico após o episódio de urgência. A procura por atendimento odontológico em casos de urgência deu-se preferencialmente por indivíduos com maior idade $(O R=1,014$, IC95\%=1,003-1,026). Conclusões: As urgências odontológicas foram mais frequentes entre indivíduos que não sabem ler, 0 que reflete uma iniquidade em saúde. O aumento da idade foi associado a uma maior procura por serviços odontológicos nos casos de urgência. $\mathrm{O}$ acesso de indivíduos socialmente vulneráveis na Atenção Primária deve ser priorizado, com ênfase em ações de promoção de saúde e prevenção de doenças bucais.

Palavras-chave: Serviços de saúde bucal. Uso de serviços de saúde. Assistência odontológica. Atendimento de urgência.

$$
\text { DENTAL EMERGENCIES PROFILE IN THE STATE OF PARAÍBA: AN ANALYSIS FROM PMAQ-AB }
$$

\section{ABSTRACT}

Objective: To evaluate factors associated with the search for emergency dental care in Primary Health Care in state of Paraiba. Methodology: Analytical cross-sectional study was carried out from the first external evaluation cycle microdata of the Program for Access and Quality Improvement of Primary Care (PMAQ-AB). Reported cases of dental urgency $(n=1,531)$ and the care demand after an emergency episode $(n=824)$ were assessed according to sociodemographic characteristics (gender, age, marital status, skin color, illiteracy, income and register in social benefits program "Bolsa Família". Data were analyzed using multivariate logistic regression, obtaining the odds ratio $(\mathrm{OR})$ and confidence interval $(95 \% \mathrm{Cl})$ measurements, considering $\mathrm{p}<0,05$. Results: Individuals who reported as not able to read were more likely $(O R=1.430,95 \% \mathrm{Cl}=1.113-1.836)$ to report some dental urgency. The variables age and income composed the adjusted regression model, but without significance $(p>0.05)$. Among individuals who reported dental urgency, $57.9 \%$ reported seeking dental care after the emergency episode. The search for dental care in urgent cases was preferably by older individuals $(\mathrm{OR}=1.014,95 \% \mathrm{Cl}=1.003-1.026)$. Conclusions: Dental emergencies were more frequent among individuals who cannot read, which reflects a health inequity. Incresed age was associated with greater seek for dental services in urgent cases. The access of socially vulnerable individuals in Primary Care should be prioritized, with emphasis on health promotion and oral disease prevention actions

Keywords: Dental health services. Health services use. Dental care. Urgency care.
\end{abstract}

RECEBIDO EM: 18/8/2019

MODIFICAÇÕES REQUERIDAS EM: 16/9/2019

ACEITO EM: 15/10/2019

\footnotetext{
Graduação em Odontologia (2014) e Mestrado em Saúde Pública (2018) pela Universidade Estadual da Paraíba. Especialização em Odontologia Legal pela Faculdade São Leopoldo Mandic. Fiscal do Conselho Regional da Paraíba, atuando na Delegacia Regional de Patos-PB. Tem experiência na área de Odontologia, atuando principalmente nos seguintes temas: Programa Saúde da Família, serviços de saúde bucal, acesso aos serviços de saúde, ensino e odontologia legal. ellenwg.d@gmail.com

2 Professor-adjunto do Departamento de Clínica e Odontologia Social da Universidade Federal da Paraíba. Realizou estágio de Pós-Doutorado no Programa de Pós-Graduação em Odontologia da Universidade Estadual da Paraíba (UEPB) (2015-2016). Possui Doutorado (2015) em Clínica Odontológica (Área de Concentração: Prótese Dentária) na Faculdade de Odontologia de Piracicaba (FOP-Unicamp). Realizou estágio de Doutoramento Sanduíche no Exterior (PDSE) como pesquisador visitante da Cardiff University (janeiro/2014 a janeiro/2015). Atua na linha de pesquisa de Epidemiologia do Envelhecimento e em Odontologia em Saúde Coletiva. Tem experiência na área de Clínica Odontológica, Diagnóstico Bucal, Odontologia Social e Preventiva, e Microbiologia. Pesquisador vinculado ao Grupo de Pesquisa em Odontopediatria e Clínica Integrada (GPOCI-UFPB). Temas de interesse em pesquisa: epidemiologia, envelhecimento, próteses dentárias, biofilmes orais, cárie dentária, economia da saúde. yuri.wanderley@yahoo.com.br

Mestranda em Ciências Odontológicas e Graduação em Odontologia pela Universidade Federal da Paraíba. Integrante do Grupo de Pesquisa em Odontopediatria e Clínica Integrada. Atua na área da Epidemiologia social, com ênfase em Saúde Bucal Coletiva. Tem experiência nas áreas de odontopediatria, patologia oral e fitoterapia aplicada à odontologia. aldelanyramalho@hotmail.com

${ }^{4}$ Professor-adjunto do Departamento de Clínica e Odontologia Social da Universidade Federal da Paraíba (UFPB). Doutor em Ciências da Saúde pela Universidade de Brasília (UnB). Mestre em Ciências da Saúde pela Universidade de Brasília (UnB). Graduado em Odontologia pela Universidade Federal da Paraíba (UFPB). Tem experiência no campo da Saúde Coletiva, com ênfase em políticas pública de saúde, gestão em saúde e sistemas de informação em saúde. ehglucena@gmail.com
} 


\section{INTRODUÇÃO}

A Atenção Primária à Saúde (APS) é a principal porta de entrada de usuários no Sistema Único de Saúde (SUS), consolidada por meio da Estratégia Saúde da Família (ESF). A partir do ano 2000, a inclusão das equipes de saúde bucal na ESF representou um importante passo na expansão da cobertura dos serviços de saúde bucal (CASOTI et al., 2014), o que reorientou o cuidado a partir de um conjunto de ações de promoção, proteção e recuperação da saúde (REIS; SCHERER; CARCERERI, 2015; BARROS et al., 2016).

Apesar da ampliação da cobertura e das tentativas de priorização das ações de promoção de saúde e prevenção de doenças bucais na ESF, parte da população brasileira ainda enfrenta dificuldades de acesso aos serviços de saúde bucal (NEVES; GIORDANI; HUGO, 2019; PERES et al., 2012). Historicamente, os indivíduos tendem a procurar o atendimento odontológico quando de episódios de dor, caracterizados como urgências (CASOTI et al., 2014). Este aspecto evidencia o perfil curativista e mutilador dos cuidados em saúde bucal no Brasil (BARROS et al., 2016).

$O$ relato de urgências odontológicas por parte do usuário reflete o padrão de utilização dos serviços de saúde e está diretamente relacionado à percepção das necessidades de saúde pelo indivíduo (PINHEIRO; TORRES, 2006; SANTOS et al., 2016; ALBUQUERQUE et al., 2016). A elevada demanda por urgências é indicativa de que as ações de prevenção e promoção da saúde falharam. Sendo assim, é desejável que a organização dos serviços priorize a redução das urgências a partir da reorganização do acesso e identifique características relacionadas a este fenômeno.

Com o objetivo de ampliar o acesso à Atenção Primária em Saúde e garantir um padrão de qualidade dos serviços ofertados, o Ministério da Saúde instituiu - Programa Nacional de Melhoria do Acesso e da Qualidade da Atenção Básica (PMAQ-AB) (BRASIL, 2011). Este Programa utiliza como principal ferramenta estratégica a avaliação de qualidade das equipes de saúde, sob diferentes aspectos, incluindo a saúde bucal (NEVES; GIORDANI; HUGO, 2019). Nesse sentido, o acesso aos serviços de saúde dos usuários do SUS pode ser analisado quanto a características sociodemográficas, disponibilidade de recursos, padrão de utilização do serviço, entre outros fatores.

Compreender os motivos relacionados às urgências odontológicas pode auxiliar o serviço de saúde a produzir mudanças quanto ao paradigma do cuidado e melhor organização do acesso. Este estudo teve como objetivo analisar os fatores associados à procura por atendimentos de urgência odontológicos na Atenção Primária à Saúde no Estado da Paraíba.

\section{METODOLOGIA}

\section{Caracterização do Estudo}

Foi realizado estudo quantitativo e analítico, com um desenho do tipo transversal, a partir dos microdados disponibilizados do primeiro ciclo de avaliação externa do PMAQ-AB, realizado em 2012. Os dados coletados são de domínio público, justificando assim a ausência de parecer de aprovação do estudo em Comitê de Ética.

No primeiro ciclo, o PMAQ-AB foi constituído por quatro fases, ou seja: fase de Adesão do município e Contratualização das equipes; fase de Desenvolvimento das ações, na qual ocorre a qualificação do processo de trabalho das equipes, autoavaliação e monitoramento de indicadores; fase de Avaliação Externa, na qual são verificadas in loco as condições de acesso e da qualidade dos serviços de saúde e fase de Recontratualização, com repactuação entre equipes e gestores e instituição de novos indicadores.

A fase de Avaliação Externa está organizada em três módulos, um referente aos aspectos da estrutura física, equipamentos, instrumentais e insumos; outro relacionado ao processo de trabalho por meio de entrevista com os profissionais e por último, uma entrevista com o usuário na Unidade Básica de Saúde, que avalia a satisfação e as condições de acesso e utilização de serviços de saúde (BRASIL, 2011).

Este estudo utilizou microdados do Estado da Paraíba, região Nordeste do Brasil, cujo Índice de Desenvolvimento Humano (IDH) foi estimado em 0,658 e a população calculada em 3.996.496 habitantes (IBGE, 2019). No ano da Avaliação Externa, o Estado possuía 1.246 Equipes de Saúde da Família (ESF), com percentual de cobertura de 92,9\% e 1.167 Equipes de Saúde Bucal, cobrindo $88 \%$ da população Paraibana (BRASIL, 2019a). No primeiro ciclo do PMAQ-AB, foram contratualizadas $625(50,2 \%)$ Equipes de Saúde da Família e $577(49,4 \%)$ de Saúde Bucal. Todos os preceitos nacionais (Resolução CNS/MS № 466/2012) e internacionais (Declaração de Helsinque) relacionados à ética em pesquisa envolvendo seres humanos foram respeitados. O checklist Strobe (Strengthening the Reporting of Observational Studies in Epidemiology) para estudos observacionais foi usado para ajudar na condução da pesquisa e relato dos resultados obtidos. 


\section{Coleta de Dados}

Os dados foram coletados a partir dos bancos de microdados disponibilizados na página da Secretaria de Atenção Primária em Saúde (BRASIL, 2019b), para o primeiro ciclo de Avaliação Externa do PMAQ$-A B$, realizado em 2012. Os bancos encontram-se disponibilizados para consulta e download em formato de planilhas eletrônicas. Foi utilizado o banco referente ao Módulo III - Entrevista com Usuário na Unidade Básica de Saúde.

\section{Variáveis Estudadas}

As variáveis sociodemográficas sexo, idade, estado civil, cor da pele, renda e cadastro no programa Bolsa Família foram consideradas variáveis independentes do estudo, as quais foram utilizadas para avaliar associação com as variáveis de desfecho: episódios de urgência odontológica e procura por atendimento odontológico. Um modelo teórico-conceitual foi elaborado especificamente para este estudo (Figura 1).

Figura 1 - Modelo teórico conceitual do estudo

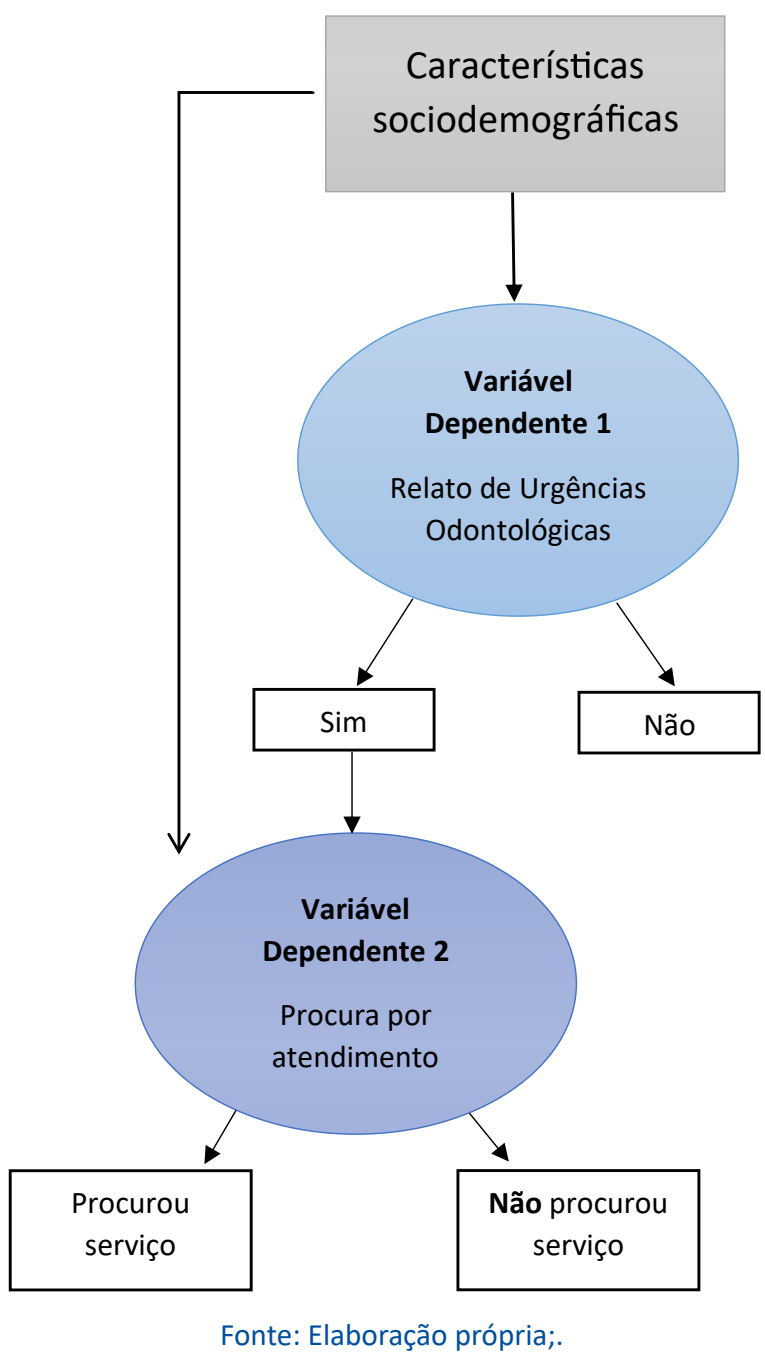

\section{Análise dos dados}

Os dados foram tabulados e analisados no software IBM SPSS Statistics (SPSS for Windows, Version 20.0. Armonk, NY: IBM Corp.). Inicialmente, realizou-se a análise estatística descritiva para caracterizar a amostra. $O$ teste qui-quadrado de Pearson $\left(\chi^{2}\right)$ foi utilizado para identificar associações entre a variável desfecho e as variáveis independentes. Posteriormente, as variáveis explicativas foram incorporadas ao modelo multivariado. Os dados foram analisados por meio de regressão logística multivariada, pela qual foram obtidas as medidas de razão de chances (OR) e intervalo de confiança (IC95\%), considerando-se $p<0,05$.

\section{RESULTADOS}

A amostra final deste estudo foi composta por 1.531 indivíduos, caracterizada por indivíduos do sexo feminino $(86,8 \%)$, que viviam com companheiro $(72,5 \%)$, de cor não branca $(69,5 \%)$, que sabiam ler $(93,3 \%)$, com cadastro no programa Bolsa Família $(61,6 \%)$ e idade variando entre 16 e 88 anos. Um total de $53,8 \%(n=824)$ relatou ter tido problemas de urgência e destes, $57,9 \%(n=477)$ procuraram atendimento de urgência. Identificou-se que 11 usuários não responderam à pergunta sobre cor da pele, 5 não mencionaram o estado civil, 2 não responderam à pergunta sobre alfabetização, e 3 não responderam se eram beneficiários do programa Bolsa Família.

A análise bivariada mostrou que relato de urgência odontológica foi associado à variável saber ler $(p=0,004)$ (Tabela 1$)$. No modelo ajustado de regressão, indivíduos que não sabiam ler apresentaram maior chance de relatar urgência odontológica ( $O R=1,430$, IC95\%=1,113-1,836) do que os indivíduos que sabiam ler. As variáveis idade e renda também compuseram o modelo, porém sem significância estatística $(p>0,05)$ (Tabela 2). 
Tabela 1 - Análise bivariada entre a variável relato de urgência odontológica e as variáveis individuais: sexo, estado civil, cor da pele, saber ler e cadastro no programa Bolsa Família

\begin{tabular}{|c|c|c|c|c|c|}
\hline \multirow[b]{3}{*}{ Variável } & \multicolumn{4}{|c|}{ Urgência } & \multirow{3}{*}{ p-valor } \\
\hline & \multicolumn{2}{|c|}{$\begin{array}{l}\text { Não teve } \\
\text { urgência }\end{array}$} & \multicolumn{2}{|c|}{$\begin{array}{c}\text { Teve } \\
\text { urgência }\end{array}$} & \\
\hline & $\mathbf{n}$ & $\%$ & $\mathbf{n}$ & $\%$ & \\
\hline \multicolumn{6}{|l|}{ Sexo } \\
\hline Masculino & 86 & 5,6 & 109 & 7,1 & \multirow{2}{*}{0,534} \\
\hline Feminino & 621 & 40,6 & 715 & 46,7 & \\
\hline \multicolumn{6}{|l|}{ Estado civil } \\
\hline Vive com companheiro & 518 & 33,9 & 605 & 39,6 & \multirow[b]{2}{*}{0,883} \\
\hline $\begin{array}{l}\text { Não vive com } \\
\text { companheiro }\end{array}$ & 189 & 12,4 & 217 & 14,2 & \\
\hline \multicolumn{6}{|l|}{ Cor da pele } \\
\hline Branca & 213 & 14,0 & 246 & 16,2 & \multirow{2}{*}{0,983} \\
\hline Não branca & 493 & 32,4 & 568 & 37,4 & \\
\hline \multicolumn{6}{|l|}{ Sabe ler } \\
\hline Sim & 610 & 39,9 & 710 & 46,4 & \multirow{3}{*}{$0,004^{*}$} \\
\hline Não & 25 & 1,6 & 54 & 3,5 & \\
\hline Só assina o nome & 72 & 4,7 & 58 & 3,8 & \\
\hline \multicolumn{6}{|c|}{ Cadastro no programa Bolsa Família } \\
\hline Sim & 471 & 30,8 & 571 & 37,4 & \multirow{2}{*}{0,250} \\
\hline Não & 235 & 15,4 & 251 & 16,4 & \\
\hline
\end{tabular}

* estatisticamente significante $(p<0,05)$

Fonte: Microdados do $1^{\circ}$ ciclo de avaliação externa do PMAQ-AB.

Tabela 2 - Fatores que determinam os episódios de urgência odontológica

\begin{tabular}{lccc}
\hline & \multicolumn{3}{c}{ Relato de urgência odontológica } \\
& Valor de $\mathbf{p}$ & $\mathbf{0 . R}$. & $\mathbf{9 5 \%}$ I.C. \\
\hline Idade & 0,100 & 0,993 & $(0,985-1,001)$ \\
Sabe ler & & & \\
Sim & 1,00 & \\
Não & $\mathbf{0 , 0 0 5 *}$ & 1,430 & $(1,113-1,836)$ \\
Renda & 0,130 & 1,000 & $(1,000-1,000)$ \\
\hline * estatisticamente significante ( $p<0,05)$ & \\
Fonte: Microdados do $1^{\circ}$ ciclo de avaliação externa do PMAQ-AB.
\end{tabular}

Dos indivíduos que relataram episódios de urgência $(n=824), 57,9 \%(n=477)$ referiram a procura por atendimento odontológico (Tabela 3). A média de idade dos indivíduos que procuraram atendimento odontológico diante de episódios de urgência foi de 47 anos, destacando-se que o aumento da idade esteve associado à maior chance de procura por atendimento em casos de urgência (OR=1,014, IC95\%=1,0031,026) (Tabela 4).
Tabela 3 - Análise bivariada entre a variável procura de atendimento odontológico após episódio de urgência e as variáveis individuais: sexo, estado civil, cor da pele, saber ler e cadastro no programa Bolsa Família

\begin{tabular}{|c|c|c|c|c|c|}
\hline \multirow[b]{3}{*}{ Variável } & \multicolumn{5}{|c|}{$\begin{array}{l}\text { Procura por atendimento odontológico } \\
\text { após episódio de urgência }\end{array}$} \\
\hline & \multicolumn{2}{|c|}{$\begin{array}{l}\text { Procurou } \\
\text { atendimento }\end{array}$} & \multicolumn{2}{|c|}{$\begin{array}{l}\text { Não procurou } \\
\text { atendimento }\end{array}$} & \multirow[t]{2}{*}{ p-valo } \\
\hline & $\mathbf{n}$ & $\%$ & $\mathbf{n}$ & $\%$ & \\
\hline \multicolumn{6}{|l|}{ Sexo } \\
\hline Masculino & 68 & 8,3 & 41 & 5,0 & \multirow[b]{2}{*}{0,307} \\
\hline Feminino & 409 & 49,6 & 306 & 37,1 & \\
\hline \multicolumn{6}{|l|}{ Estado civil } \\
\hline $\begin{array}{l}\text { Vive com } \\
\text { companheiro }\end{array}$ & 352 & 42,8 & 253 & 30,8 & \multirow{2}{*}{0,701} \\
\hline $\begin{array}{l}\text { Não vive com } \\
\text { companheiro }\end{array}$ & 123 & 15,0 & 94 & 11,4 & \\
\hline \multicolumn{6}{|l|}{ Cor da pele } \\
\hline Branca & 138 & 17,0 & 108 & 13,3 & \multirow{2}{*}{0,533} \\
\hline Não branca & 332 & 40,8 & 236 & 29,0 & \\
\hline \multicolumn{6}{|l|}{ Sabe ler } \\
\hline Sim & 442 & 53,6 & 326 & 39,7 & \multirow{2}{*}{0,609} \\
\hline Não & 33 & 4,0 & 21 & 2,6 & \\
\hline \multicolumn{6}{|c|}{ Cadastro no programa Bolsa Família } \\
\hline Sim & 342 & 41,7 & 228 & 27,7 & \multirow{2}{*}{$0,046^{*}$} \\
\hline Não & 132 & 16,1 & 119 & 14,5 & \\
\hline
\end{tabular}

Tabela 4 - Fatores que determinam a procura por atendimento odontológico após episódios de urgência odontológica

\begin{tabular}{|c|c|c|c|c|}
\hline & \multicolumn{4}{|c|}{$\begin{array}{l}\text { Procura de serviço em caso de } \\
\text { urgência odontológica }\end{array}$} \\
\hline & $\begin{array}{l}\text { Valor } \\
\text { de } p\end{array}$ & O.R & $95 \%$ & ' I.C. \\
\hline \multicolumn{5}{|l|}{ Sexo } \\
\hline Masculino & & 1,00 & & \\
\hline Feminino & 0,138 & 1,178 & 0,949 & 1,461 \\
\hline Idade & $0,013^{*}$ & 1,014 & 1,003 & 1,026 \\
\hline \multicolumn{5}{|c|}{ Cadastro no programa Bolsa Família } \\
\hline Sim & & 1,00 & & \\
\hline Não & 0,100 & 1,140 & 0,975 & 1,332 \\
\hline
\end{tabular}

* estatisticamente significante $(p<0,05)$

Fonte: Microdados do $1^{\circ}$ ciclo de avaliação externa do PMAQ-AB.

\section{DISCUSSÃO}

Apesar dos avanços obtidos na ampliação da oferta de assistência odontológica na atenção primária (PALACIO et al., 2014), as urgências odontológicas 
ainda podem ser consideradas problema de saúde pública, devido a sua morbidade e impacto na qualidade de vida (MARTINS et al., 2014). A análise das urgências revela o padrão de utilização dos serviços de saúde bucal e pode contribuir para mudanças no processo de trabalho do cuidado em saúde.

Os eventos mais frequentemente relacionados à urgência odontológica são dores de origem pulpar, patologias periapicais, cáries profundas, pericoronarite e fraturas dentárias (ESTRELA et al., 2011; MARTINS et al., 2014; ALBUQUERQUE et al., 2016). Um percentual significativo de indivíduos neste estudo relatou ter tido episódios de urgência e que procurou atendimento na própria Unidade de Saúde. As diretrizes da Política Nacional de Saúde Bucal determinam que os casos de urgência devem ser priorizados nos serviços de saúde, como forma de garantir o acesso universal à assistência odontológica (MATSUMOTO et al., 2017).

As desigualdades no acesso aos serviços odontológicos podem gerar uma demanda reprimida que, consequentemente, aumentará a procura por serviços de urgência, na maioria dos casos motivada por dores de origem dentária (ALBUQUERQUE et al., 2016). Importante salientar que a inserção de equipes de saúde bucal na atenção primária visava a mudanças no modelo de atenção e reorganização das práticas de saúde bucal. Durante os episódios de urgência, porém, há uma maior procura por procedimentos curativos, conservando o modelo assistencial curativista (BARROS et al., 2016).

Assim, uma maior procura por atendimento de urgência pode significar desvio de finalidade dos serviços, uma vez que as ações de promoção de saúde e prevenção de agravos deveriam ser efetivas e evitar que os problemas de saúde bucal se instalassem ou evoluíssem para situações mais complexas, ocasionando as urgências. Uma análise dos resultados do SB-Brasil 2003 demonstrou que adultos em piores condições de saúde utilizaram com maior frequência os serviços públicos (PINTO; MATOS; LOYOLA FILHO, 2012). Além disso, maior demanda por atendimento de urgência entre os setores mais vulneráveis da população, como baixo nível de escolaridade e renda, tem sido observada em outras regiões do Brasil (ROJAS et al., 2015; GUSKUMA et al., 2017; MATSUMOTO et al., 2017).

Embora o modelo multivariado deste estudo não tenha demonstrado o efeito do Programa Bolsa Família na procura por serviços odontológicos em casos de urgência, há de se considerar que beneficiários de programas afirmativos do governo são, em geral, indivíduos expostos à maior vulnerabilidade, menor renda e escolaridade, o que justifica os resultados encontrados.

A escolaridade de um indivíduo pode influenciar diretamente no seu nível de saúde. Por sua vez, um maior grau de instrução pode ter relação direta com comportamentos positivos em saúde bucal, uma vez que estas pessoas procuram mais o serviço para consultas de prevenção e acompanhamento (PANDOLFI; BARCELLOS; MIOTTO, 2006; PINTO; MATOS; LOYOLA FILHO, 2012). Por outro lado, indivíduos em situação de maior vulnerabilidade social, como os analfabetos e de menor escolaridade, buscam o serviço por problemas autopercebidos, sendo mais frequentemente submetidos a tratamentos curativos (GUSKUMA et al., 2017). As iniquidades em saúde, com a presença de restrição de acesso, dificultam as oportunidades de tratamento preventivo ou precoce para indivíduos socialmente vulneráveis, aumentando a chance de episódios de urgências nesse grupo social (CASSAL; CARDOSO; BAVARESCO, 2011).

A procura por serviços de urgência é maior em faixas etárias entre 18 e 35 anos e, em faixas etárias maiores que esta, a procura por esses serviços diminui. Esse fato pode estar relacionado ao edentulismo nessa faixa etária, com consequente redução na experiência de cárie e diminuição dos problemas odontológicos (QUEIROZ et al., 2019). Além disso, historicamente as estratégias e modelos de cuidado são voltados para crianças e adolescentes em idade escolar, favorecendo uma maior prevalência de episódios de urgência na população adulta (LIMA et al., 2013).

Um estudo realizado no município de Porto Alegre, região Sul do país, demonstrou que a média de idade de indivíduos que procuraram atendimento de urgência foi de 44 anos (CASSAL; CARDOSO; BAVARESCO, 2011), corroborando os achados do presente estudo. Apesar de este trabalho não ter demonstrado diferença significativa entre diferentes idades e o relato de urgência odontológica, foi observada uma maior utilização do serviço público odontológico por indivíduos de mais idade nos casos de urgência. A transição demográfica ocorrida no país foi caracterizada por um aumento significativo na população idosa e expectativa de vida e essa população possui demandas próprias em saúde bucal que necessitam de um direcionamento do cuidado para atender às suas necessidades (FONSECA; FONSECA; MENEGHIM, 2017).

Devido à natureza transversal deste estudo, é importante levar em consideração a possibilidade de causalidade reversa na explicação dos achados. Além disso, pelo fato de as entrevistas serem realizadas 
com usuários na própria unidade de saúde, os achados podem ser enviesados pela realidade socioepidemiológica local. Ainda que tenha sido considerada a análise dos usuários do Estado da Paraíba, este instrumento foi aplicado em todos os Estados do Brasil, possibilitando comparar diferentes cenários em um estudo futuro. Embora a frequência seja irrelevante e tenha baixo potencial de interferir nos resultados, a perda de algumas informações por não resposta dos usuários deve ser destacada como possível limitação de estudos epidemiológicos.

É importante ressaltar que a maioria dos estudos que tratam dos fatores relacionados ao relato de urgência odontológica e o acesso aos serviços de saúde bucal abordam grupos específicos, como escolares ou idosos. São poucas as pesquisas que incluem faixas etárias de adultos jovens e adultos. Apesar de o Estado da Paraíba ser de pequeno porte, os achados permitem discutir um tema relevante para orientação das práticas de cuidado em saúde bucal e assistência na ESF, sendo representativo da população de referência.

\section{CONSIDERAÇÕES FINAIS}

As urgências odontológicas foram mais frequentes entre indivíduos que não sabem ler, enquanto o aumento da idade foi associado a uma maior procura por serviços odontológicos nos casos de urgência. Diante dos achados deste estudo, destaca-se o papel marcante do baixo nível educacional como iniquidade em saúde, possuindo reflexos negativos na condição bucal dos usuários. $O$ acesso de indivíduos socialmente vulneráveis na atenção primária deve ser priorizado, sobretudo em relação a ações de promoção de saúde e prevenção de doenças bucais.

\section{REFERÊNCIAS}

ALBURQUERQUE, Y. E.; ZUANON, A. C. C.; PANSANI, C. A.; GIRO, E. M. A.; LIMA, F. C. B. A.; PINTO, L. A. M. S.; CORDEIRO, R. C. L.; COSTA, J. H.; BRIGHENTI, F. L. Perfil do atendimento odontológico no Serviço de Urgência para crianças e adolescentes da Faculdade de Odontologia de Araraquara (FOAr) - Unesp. Rev Odontol. Unesp, v. 45, n. 2, p. 115-120, 2016.

BARROS, S. G.; CANGUSSU, M. C. T.; CRUZ, D. N.; SILVA, L. O. R.; RONCALLI, A. G. Impacto da implantação das Equipes de Saúde Bucal na Estratégia Saúde da Família em dois municípios do Estado da Bahia. Rev. Saúde Colet. UEFS, v. 6, n. 2, p. 37-42, 2016.

BRASIL. Ministério da Saúde. Programa Nacional de Melhoria do Acesso e da Qualidade da Atenção Básica. Portaria de no 1654/2011. Brasília, DF. 2011.
BRASIL. Ministério da Saúde. Secretaria de Atenção Primária à Saúde. Departamento de Saúde da Família. 2019a. Histórico de Cobertura de Equipes de Saúde Bucal na Estratégia de Saúde da Família. Disponível em: https://egestorab. saude.gov.br/. Acesso em: 13 maio 2019.

BRASIL. Ministério da Saúde. Secretaria de Atenção Primária à Saúde. Ações, programas e estratégias. 2019b. Programa Nacional de Melhoria do Acesso e da Qualidade. Disponível em: https://aps.saude.gov.br/ape/pmaq. Acesso em: 10 jul. 2019.

CASOTTI, E.; CONTARATO, P. C.; FONSECA, A. B. M.; BORGES, P. K. O.; BALDANI, M. H. Atenção em Saúde Bucal no Brasil: uma análise a partir da Avaliação Externa do PMAQ-AB. Saúde Debate, Londrina, v. 39, n. esp, p. 140-157, 2014.

CASSAL, J. B.; CARDOZO, D. D.; BAVARESCO, C. S. Perfil dos usuários de urgência odontológica em uma Unidade de Atenção Primária à Saúde. Rev. APS, Juiz de Fora, v. 14, n. 1, p. 85-92, 2011.

ESTRELA, C.; GUEDES, A. O.; SILVA, J. A.; LELES, C. R.; ESTRELA, C. R. A.; PÉCORA, J. D. Diagnostic and Clinical Factors Associated with Pulpal and Periapical Pain. Braz. Dent. j., Ribeirão Preto, v. 22, n. 4, p. 306-311,2011.

FONSECA, E. P.; FONSECA, S. G. O.; MENEGHIM, M. C. Fatores associados ao uso dos serviços odontológicos por idosos residentes no Estado de São Paulo, Brasil. Rev. Bras. Geriatr. Gerontol., Rio de Janeiro, v. 20, n. 6, p. 790-801, 2017.

IBGE. Instituto Brasileiro de Geografia e Estatística. 2019. Panorama da Paraíba. Disponível em: https://www.ibge. gov.br/cidades-e-estados/pb/.html? Acesso em: 15 jul. 2019.

GUSKUMA, R. C.; LAGES, V. A.; HAFNER, M. B.; RANDO-MEIRELLES, P. M.; CYPRINAO, S.; SOUSA, M. L. R.; BATISTA, M. J. Fatores associados à prevalência e intensidade de odontalgia em crianças de municípios da região de Campinas, São Paulo. Rev. Paul. Pediatr., São Paulo, v.35, n.3, p.322-330. 2017.

LIMA, L. S.; SILVA, K. M. G.; MELLO, M. V. F. M.; SANTOS, M. S.; ARAÚJO, M. S.; CANGUSSU, M. C. T. Condição de urgência odontológica e fatores associados em trabalhadores da construção civil - Bahia, 2008. Rev. Odontol. Unesp., Araraquara, v. 42, n. 1, p. 48-53, 2013.

MARTINS, E. P.; OLIVEIRA, O. R; BEZERRA, S. R. S.; DOURADO, A. T. Estudo epidemiológico de urgências odontológicas da FOP/UPE. RFO UPF, Passo Fundo, v. 19, n. 3, p. 316-322, 2014.

MATSUMOTO, M. S. A.; GATTI, M. A. N.; CONTI, M. H. S.; SIMEÃO, S. F. A. P.; FRANZOLIN, S. O. B.; MARTA, S. N. Determinants of Demand in the Public Dental Emergency Service. J Contemp Dent Pract, Nova Delhi, v. 18, n. 2, p. 156-161, 2017.

NEVES, M.; GIORDANI, J. M. A.; HUGO, F. N. Atenção primária à saúde bucal no Brasil: processo de trabalho das equipes de saúde bucal. Ciênc. Saúde Colet., Rio de Janeiro, v. 24, n. 5, p. 1.809-1.820, 2019. 
PALACIO, D. C.; VAZQUEZ, F. L.; RAMOS, V. R.; PERES, S. V.; PEIREIRA, A. C.; GUERRA, L. M.; CORTELLAZZI, K. L.; BULGARELI, J. V. Evolução de indicadores pós-implantação da saúde bucal na Estratégia Saúde da Família. Einstein, São Paulo, v. 12, n. 3, p. 274-281, 2014.

PANDOLFI, M.; BARCELlOS, L. A.; MIOTTO, M. H. M. B. Perfil dos usuários e motivo da procura pelos serviços odontológicos das unidades de saúde de Vitória - ES. Ufes Rev. Odontol., Vitória, v. 8, n. 2, p. 37-44, 2006.
PERES, K. G.; PERES, M. A.; BERTOLDI, A. D.; BASTOS, J. L.; BARROS, A. J. D. Redução das desigualdades sociais na utilização de serviços odontológicos no Brasil entre 1998 e 2008. Rev. Saúde Pública, São Paulo, v. 46, n. 2, p. 250-258, 2012.

PINHEIRO, R. S.; TORRES, T. Z. G. Uso de serviços odontológicos entre os Estados do Brasil. Ciênc. Saúde Colet., Rio de Janeiro, v. 11, n. 42, p. 999-1.010, 2006.

PINTO, R. S.; MATOS, D. L.; LOYOLA FILHO, A. I. Características associadas ao uso de serviços odontológicos públicos pela população adulta brasileira. Ciênc. Saúde Colet., Rio de Janeiro, v. 17, n. 2, p. 531-544, 2012.

QUEIROZ, M. F.; VERLI, F. D.; MARINHO, S. A.; PAIVA, P. C. P.; SANTOS, S. M. C.; SORES, J. A. Dor, ansiedade e qualidade de vida relacionada à saúde bucal de pacientes atendidos no serviço de urgência odontológica. Ciênc. Saúde Colet., Rio de Janeiro, v. 24, n. 4, p. 1.277-1.286, 2019.

REIS, W. G.; SCHERER, M. D. A.; CARCERERI, D. L. O trabalho do cirurgião-dentista na Atenção Primária à Saúde: entre o prescrito e o real. Saúde Debate, Londrina, v. 39, n. 104, p. 56-64, 2015.

ROJAS, G. C. S.; VAZQUEZ, F. L.; BULGARELI, J. V.; MENEGHIM, M. C.; PEREIRA, A. C. Influence of scheduling in clinical dental care on indicators of oral health. $R G O$, Porto Alegre, v. 63, n. 3, p. 283-290, 2015.

SANTOS, L. M.; NORO, L. R. A.; RONCALLI, A. G.; TEIXEIRA, A. K. M. Autopercepção sobre saúde bucal e sua relação com utilização de serviços e prevalência de dor de dente. Rev. Ciênc. Plur., Natal, v. 2, n. 2, p. 14-27, 2016. 